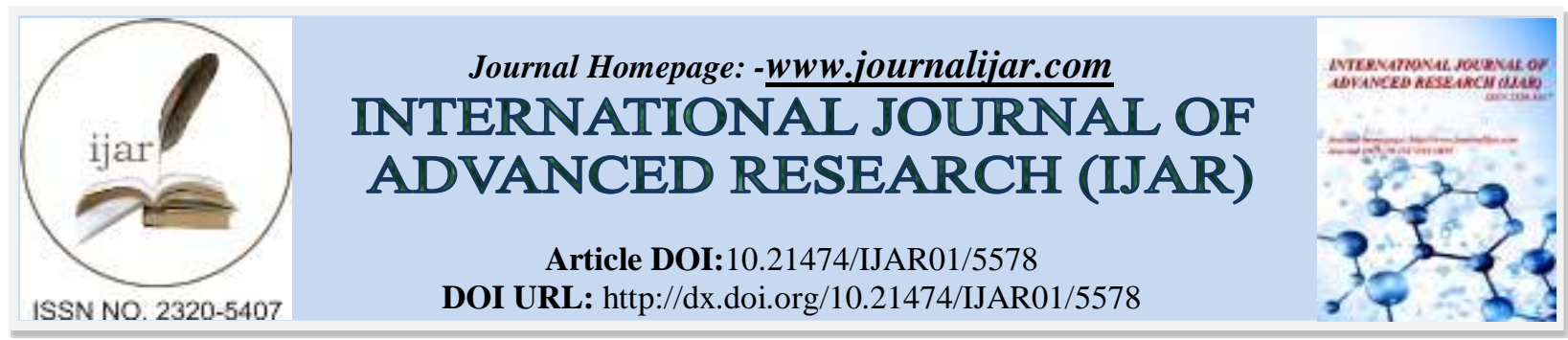

RESEARCH ARTICLE

\title{
EFFECT OF IN VITRO ACTIVATION BY GLUTATHIONE SUPPLEMENTED MEDIUM ON SPERM MOTILITY, MORPHOLOGY AND VITALITY DURING SPERM CRYOPRESERVATION OF ASTHENOZOOSPERMIC MEN.
}

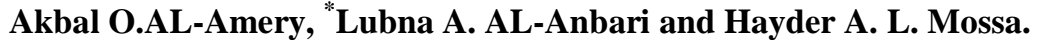

High Institute for Infertility Diagnosis and Assisted Reproductive Technologies, Al-Nahrain University, BaghdadIRAQ.
\end{abstract}

\section{Manuscript Info \\ Manuscript History \\ Received: 09 August 2017 \\ Final Accepted: 11 September 2017 \\ Published: October 2017}

Key words:-

glutathione, cryopreservation, asthenozoospermic men.

\begin{abstract}
Background: Sperm cells from asthenozoospermia have humble motility, Glutathione is added in the medium to increase sperm motility and improve morphologically normal sperm. Cryopreservation of human spermatozoa is one of the best active and acceptable methods to preserve male fertility. However, cryopreservation may lead to changes in sperm structure and decline in sperm functional parameters.

Objective: This study has been designed to investigate the effect of Glutathione on certain sperm functional parameters in Asthenozoospemic men before and after cryopreservation. Materials and Methods:The study included forty five asthenozoospermic infertile men. Ejaculated semen were obtained from patients and divided in to two equal portions, first portion was activated by free FertiCult Flushing medium as (control), second portion activated by Glutathione(GSH) in $5 \mathrm{mM}$ concentration. The samples were cryopreserved in liquid nitrogen for one month. All the samples examined for sperm motility, morphology and vitality before and after cryopreservation-thawing, and comparison in these parameters between the two groups done.

Results: In vitro activation by GSH resulted in significant $(\mathrm{P}<0.05)$ decrease in sperm concentration, highly significant increase $(\mathrm{P}<0.001)$ in sperm motility, highly significant decrease in immotile sperm (grade D) and significant increase $(\mathrm{P}<0.05)$ in morphologically normal sperm(MNS) compared with pre activation. After cryopreservationthawing, there was significant difference $(\mathrm{P}<0.05)$ in certain sperm functional parameters compared with control.

Conclusion: There is positive effect and improvement in certain sperm functional parameters after in vitro sperm activation by GSH supplemented medium, before and after cryopreservation.
\end{abstract}

Copy Right, IJAR, 2017,. All rights reserved.

\section{Introduction:-}

World Health Organization (WHO) considers infertility as an imperative issue of reproductive health. Approximately 10 to 15 percent of couples are infertile. In about half of those cases male infertility plays a role $^{(1)}$ Sperm must have adequate motility in order to penetrate both the layers ofcoronal cells and the zona pellucida

*Corresponding Author:-Lubna A. AL-Anbari.

Address:-High Institute for Infertility Diagnosis and Assisted Reproductive Technologies , Al-Nahrain 
before fusing with egg's cell membrane (Oolemma) ${ }^{(2)}$, motility reflects sperm functional capacity, and considered a valuable indicator of a man's fertilization potential ${ }^{(3)}$.Several studies have proved the correlation of motility with the fertilization rate in vivo and in vitro, therefore asthenozoospermia is one of the major causes of infertility or reduced fertility in men ${ }^{(4)}$.

Semen cryopreservation is one of the most active and acceptable methods to preserve male fertility potential and considered a routine procedure in assisted reproductive technologies (ART's) ${ }^{(5)}$.However Cryopreservation has negative effects on sperm motility and decreases the normal ratio of sperm vital parameters after thawing compared with the pre-cryopreservation step ${ }^{(6)}$. That may be a result of reduced glycocalyx (cell-surface carbohydrates) due to increment in hydroxyl $(\mathrm{OH})$ and superoxide $(\mathrm{O} 2-)$ radicals ${ }^{(7)}$. The antioxidant supplementation in freezing extender like Glutathione, which classified as antioxidant has been used to define against free radicals ${ }^{(8)}$. Glutathione (GSH) is a thioltripeptide (yglutamylcysteinyl glycine) with several biological functions found widely in animal body, in somatic cells and also in gametes. This thiol has an important role in anti-oxidation process of endogenous and exogenous composts ${ }^{(9)}$. Glutathione is a little protein, delivered normally in the body, and is quickly devoured during any sort of stress, fatigue and even exercise ${ }^{(10)}$. GSH has several additional functions in cells such as storage and transport of nitric oxide, participates in metabolism of estrogens and prostaglandins, act as a reserve form of cysteine, and carrier of an active thiol group in the form of a cysteine residue ${ }^{(11)}$. GSH plays role in reduction of ribonucleotides to deoxyribonucleotides and the maturation of iron-sulfur clusters of diverse proteins. GSH involved in the operation of certain transcription factors, and detoxification of many endogenous compounds and xenobiotics $^{(12)}$.

Glutathione has been detected in various concentrations in male reproductive tissues in different species including human being. Substantial quantities of GSH are found in the testis, reproductive tract fluids and epididymal spermatozoa ${ }^{(13)}$.

\section{Materials and Methods:-}

This study was carried out in the High Institute for Infertility Diagnosis and Assisted Reproductive Technologies/ Al-Nahrain University, The study were included forty five asthenozoospermic subjects .Semen samples were collected by masturbation into wide-mouth containers, after 3-7 days of sexual abstinence, immediately placed in an incubator at $37 \mathrm{C}^{\circ}$ till complete liquefaction. After liquefaction, the semen samples were analyzed by macroscopic and microscopic examinations according to standard criteria of WHO 1999 and 2010.

Assessment of Sperm Motility: Sperm motility was examined by microscope. To evaluate this parameter, one drop (10 $\mathrm{\mu l}$ ) of semen was placed on a slide and spermatozoa with progressive motility counted using X40 objective lens. At least one hundred spermatozoa were counted, and the number of progressively motile and immotile sperm was documented, according to WHO 1999.

Assessment of Sperm Morphology: Examination of morphologically normal sperm was performed by Hematoxylin-Eosin stain. Semen samples were evaluated by placing $5 \mu 1$ on slide, thin smear made, and dried in air at room temperature.Fixed and stained by Hematoxylin-Eosin stain. At least 200 sperm were scored on randomly chosen fields, under oil immersion with high resolution (X100) objective lens.

Assessment of Sperm Vitality: This is a method used to distinguish live non motile sperm from dead sperm ${ }^{(26) .}$ Sperm vitality was assessed by mixture of Eosin Y $(0.5 \%)$-Nigrosin $(0.1 \%)$ stain. Dead sperm were stained by Eosin, while live sperm reserved their cellular membrane integrity preventing the stain from entering cells. Nigrosin served as a background stain. Unstained spermatozoa which appeared white were classified as "live", and those that show any pink or red color were consider "dead".

Preparation for in vitro sperm activation:After liquefacation of ejaculated samples, centrfiugation-swim up activation technique performed, each sample was divided into two portions, one portion was activation by free FertiCult Flushing medium, and second portion was activated by Glutathione supplemented medium.

Preparation of Glutathione (GSH) Solution: Glutathione was prepared in concentration of $5 \mathrm{mM}^{(14)}$, by adding $15.4 \mathrm{mg}$ of Glutathione poder to $10 \mathrm{ml}$ of FertiCult Flushing medium. After well shaking, filtered by using millipore $0.45 \mu \mathrm{M}^{(15)}$ and fixed at 7.4-7.8 $\mathrm{pH}$ at room temperature $\left(25 \mathrm{C}^{\circ}\right)$, and sterilized by UIV light. 
Process of cryopreservation: The samples were frozen in liquid nitrogen $\left(\mathrm{LN}_{2}\right)$ at $\left(-196^{\circ} \mathrm{C}\right)$ in a formal Lab Dewar Tank, $1 \mathrm{ml}$ of sample was transferred to a cryovial, then $0.7 \mathrm{ml}$ of sperm freeze medium (drop by drop) within 5 minutes was added. The cryovials was placed in a cryoholders and kept in refrigerator for 15 minutes, then the cryoholders exposed to liquid nitrogen vapor for 10 minutes, before they immersed and kept in liquid nitrogen $\operatorname{tank}^{(16)}$.

Process of Thawing: The samples were thawed after one month, by transferring the cryovials from liquid nitrogen to room temperature for 10 minutes, then to a water bath at $37^{\circ} \mathrm{C}$ until ice melting within $5-10$ minutes ${ }^{(17)}$. The vials were diluted with $2 \mathrm{ml}$ of FertiCult Flushing medium and centrifuged at $2500 \mathrm{rpm}$ for 5 minutes. Supernatant was discarded and $0.5 \mathrm{ml}$ of FertiCult Flushing was added.

Statistical Analysis: The Statistical Analysis System-SAS (2016) program was used to measure the effect of different factors in study parameters. Least significant difference-LSD test (ANOVA) was used for significant compare between means ${ }^{(18)}$. Differences between values were considered significant at $\mathrm{P}<0.05$ and high significant at $\mathrm{P}<0.001$.

\section{Results:-}

Effect of in vitro activation by Free FertiCult medium (control) on certain sperm function parameters:

Mean sperm concentration (million/ml) after in vitro activation using FertiCult medium only (control), showed highly significant decrement $(\mathrm{P}<0.001)$ compared to the result before activation. There was no significant difference in active sperm motility grade $A$. There was significant $(\mathrm{p}<0.05)$ increase in grade $\mathrm{B}$, grade $\mathrm{A}+\mathrm{B}$ and $\mathrm{Grade} \mathrm{C}$ compared to semen before activation. There was high significant $(\mathrm{p}<0.001)$ decrease in immotile sperm grade $\mathrm{D}$ compared with fresh semen. There was significant $(\mathrm{P}<0.05)$ increment in percentage of morphologically normal sperm (MNS) following activation with FertiCult medium as shown in table (1).

Effect of in vitro activation by medium containing Glutathione (GSH) on certain sperm function parameters: Mean sperm concentration (million/ml) after in vitro activation using FertiCult medium supplemented with Glutathione showed highly significant decrement $(\mathrm{P}<0.001)$, compared to the result before activation .There was a highly significant $(\mathrm{P}<0.001)$ increase in active sperm motility grade $\mathrm{A}$, grade $\mathrm{B}$ and grade $(\mathrm{A}+\mathrm{B})$ activated by Glutathione compared to fresh semen, and control medium. There was highly significant $(\mathrm{P}<0.001)$ decrease in the immotile sperm (grade D) compared with fresh semen . The percentage of morphologically normal sperm (MNS)was a significantly $(\mathrm{P}<0.05)$ increase compared to the fresh semen and control medium. As shown in table (1).

Table 1:- Certain sperm function parameters after in vitro activation by free control medium and Glutathione supplemented medium using centrifugation-swim up technique and before cryopreservation

\begin{tabular}{|c|l|l|l|l|}
\hline \multicolumn{2}{|c|}{$\begin{array}{c}\text { Certain sperm function } \\
\text { parameters }\end{array}$} & \multicolumn{1}{|c|}{$\begin{array}{c}\text { Before activation } \\
\text { (fresh semen) } \\
\text { M } \pm \text { S.E }\end{array}$} & $\begin{array}{c}\text { After Activation } \\
\text { By FertiCult medium } \\
\text { only(control) } \\
\text { M } \pm \text { S.E }\end{array}$ & $\begin{array}{c}\text { After Activation } \\
\text { ByGlutathione } \\
\text { M } \pm \text { S.E }\end{array}$ \\
\hline Sperm concentration (Million/ml) & $52.73 \pm 1.89 \mathrm{a}$ & $23.48 \pm 0.91 \mathrm{~b}$ & $28.73 \pm 0.90 \mathrm{~b}$ \\
\hline $\begin{array}{c}\text { Progressive sperm } \\
\text { motility (\%) }\end{array}$ & GradeA & $0.22 \pm 0.15 \mathrm{~d}$ & $2.75 \pm 0.43 \mathrm{c}$ & $7.62 \pm 0.77 \mathrm{bc}$ \\
\cline { 2 - 5 } & GradeB & $33.28 \pm 1.08 \mathrm{c}$ & $40.00 \pm 0.88 \mathrm{bc}$ & $48.73 \pm 1.07 \mathrm{ab}$ \\
\cline { 2 - 5 } & GradeA+B & $33.51 \pm 1.14 \mathrm{~b}$ & $42.75 \pm 1.14 \mathrm{~b}$ & $56.35 \pm 1.54 \mathrm{a}$ \\
\hline Immotile sperm (\%) & Grade D & $37.82 \pm 1.39 \mathrm{a}$ & $17.35 \pm 1.06 \mathrm{~b}$ & $3.40 \pm 0.49 \mathrm{c}$ \\
\hline \multicolumn{2}{|c|}{ Morphologically Normal Sperm (\%) } & $30.06 \pm 1.39 \mathrm{c}$ & $35.00 \pm 0.50 \mathrm{~b}$ & $40.00 \pm 0.65 \mathrm{ab}$ \\
\hline
\end{tabular}

$\mathrm{M} \pm \mathrm{SE}=$ Mean \pm Standard Error, Different letters mean significant

Same letters mean non-significant Number of asthenozoospermia $=45$

Effect of in vitro activation by free medium (control) and GSH supplemented medium on certain sperm function parameters of cryopreserved-thawed semen of asthenozoospermic men:

The results of sperm parameters of in vitro activation by free medium and GSH after freezing- thawing was significant decrease $(\mathrm{P}<0.05)$ in sperm concentration after cryopreservation-thawing compared to the result before cryopreservation. Besides, there were significant $(\mathrm{P}<0.05)$ difference in means of sperm concentration between them. The highest concentration observed when using GSH supplemented medium, There was significant $(\mathrm{P}<0.05)$ decrease in active sperm motility grade A, after thawing compared to pre-cryopreservation. From the other hand, active sperm motility grade $\mathrm{B}$ was highly significant $(\mathrm{P}<0.001)$ increased when using GSH supplemented medium 
compared to control medium. The results after freezing-thawing was highly significant difference $(\mathrm{P}<0.001)$ in sperm motility grad A+B in GSH supplemented medium compared to control medium. There was highly significant $(\mathrm{P}<0.001)$ increase in immotile sperm (grade $\mathrm{D})$ recorded after cryopreservation- thawing compared to precryopreservation, and there was a significance between control and GSH supplemented media. Cryopreservation causes significant $(\mathrm{P}<0.05)$ decrease in percentage of $(\mathrm{MNS})$ compared to pre cryopreservation, also there was significant decrease in control medium compared GSH supplemented medium. Vitality test was done post sperm thawing accordingly to criteria that mentioned before, as shown in figure $(1,2)$, and the results showed that there was high significant $(\mathrm{P}<0.001)$ increment in GSH supplemented medium compared with control medium, as shown in table (2).

Table (2):- Certain sperm function parameters after in vitro activation, and after cryopreservation by free control medium and Glutathione supplemented medium using centrifugation-swim up technique:

\begin{tabular}{|c|l|l|l|}
\hline \multicolumn{2}{|c|}{$\begin{array}{c}\text { Certain sperm function } \\
\text { parameters }\end{array}$} & \multicolumn{1}{c|}{$\begin{array}{c}\text { After cryopreservation- } \\
\text { Thawing of FertiCult medium only(control)M } \pm \text { S.E }\end{array}$} & $\begin{array}{c}\text { After cryopreservation- } \\
\text { Thawing of Glutathione } \\
\text { M } \pm \text { S.E }\end{array}$ \\
\hline Sperm concentration (Million/ml) & $16.20 \pm 0.60 \mathrm{~b}$ & $21.82 \pm 0.66 \mathrm{a}$ \\
\hline Progressive sperm motility (\%) & GradeA & $0.00 \pm 0.00 \mathrm{~b}$ & $2.77 \pm 0.42 \mathrm{~b}$ \\
\cline { 2 - 4 } & GradeB & $18.17 \pm 0.87 \mathrm{c}$ & $33.00 \pm 0.86 \mathrm{ab}$ \\
\cline { 2 - 4 } & GradeA+B & $18.17 \pm 0.87 \mathrm{c}$ & $35.82 \pm 1.05 \mathrm{~b}$ \\
\hline Immotile sperm (\%) & Grade D & $61.88 \pm 1.19 \mathrm{a}$ & $40.06 \pm 1.00 \mathrm{~b}$ \\
\hline \multicolumn{2}{|c|}{ Morphologically Normal Sperm (\%) } & $23.95 \pm 0.58 \mathrm{c}$ & $33.66 \pm 0.56 \mathrm{ab}$ \\
\hline \multicolumn{2}{|c|}{ Vitality sperm (\%) } & $42.62 \pm 0.92 \mathrm{c}$ & $64.73 \pm 0.94 \mathrm{~b}$ \\
\hline
\end{tabular}

$\mathrm{M} \pm \mathrm{SE}=$ Mean \pm Standard Error Different letters mean a significant

Same letters mean non-significant Number of asthenozoospermia $=45$

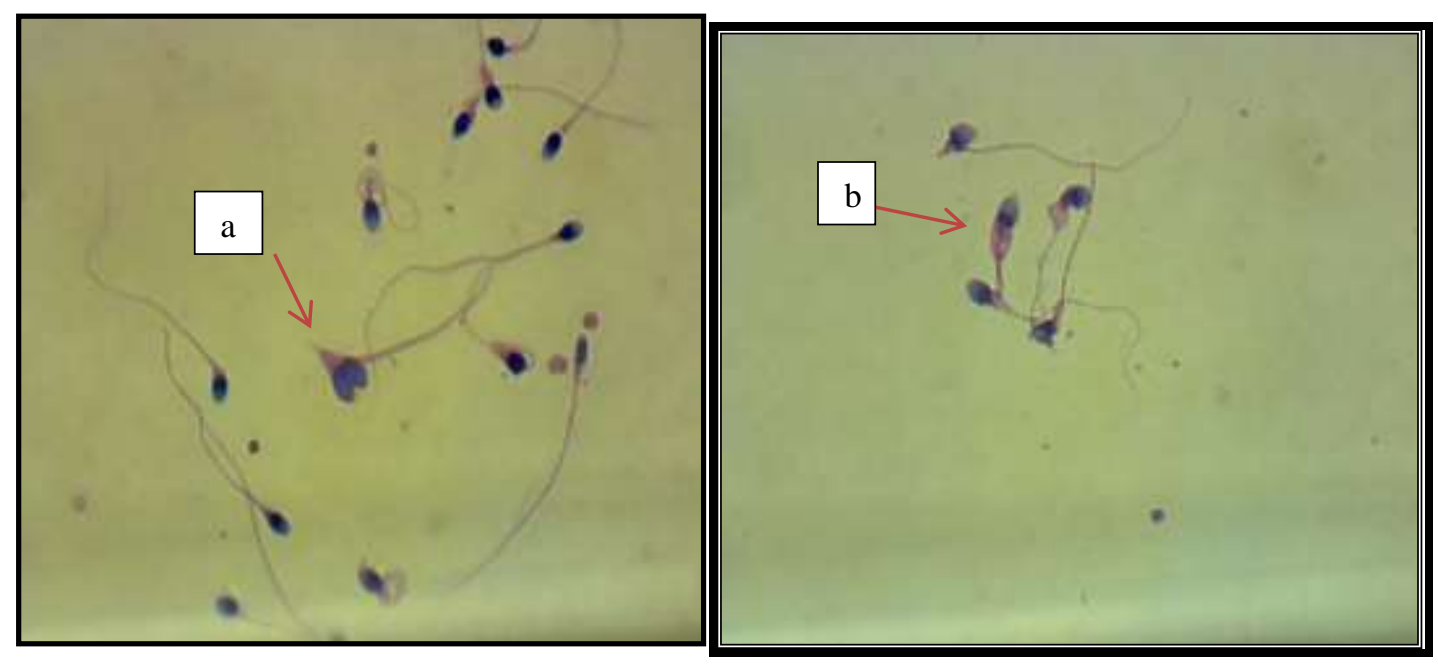




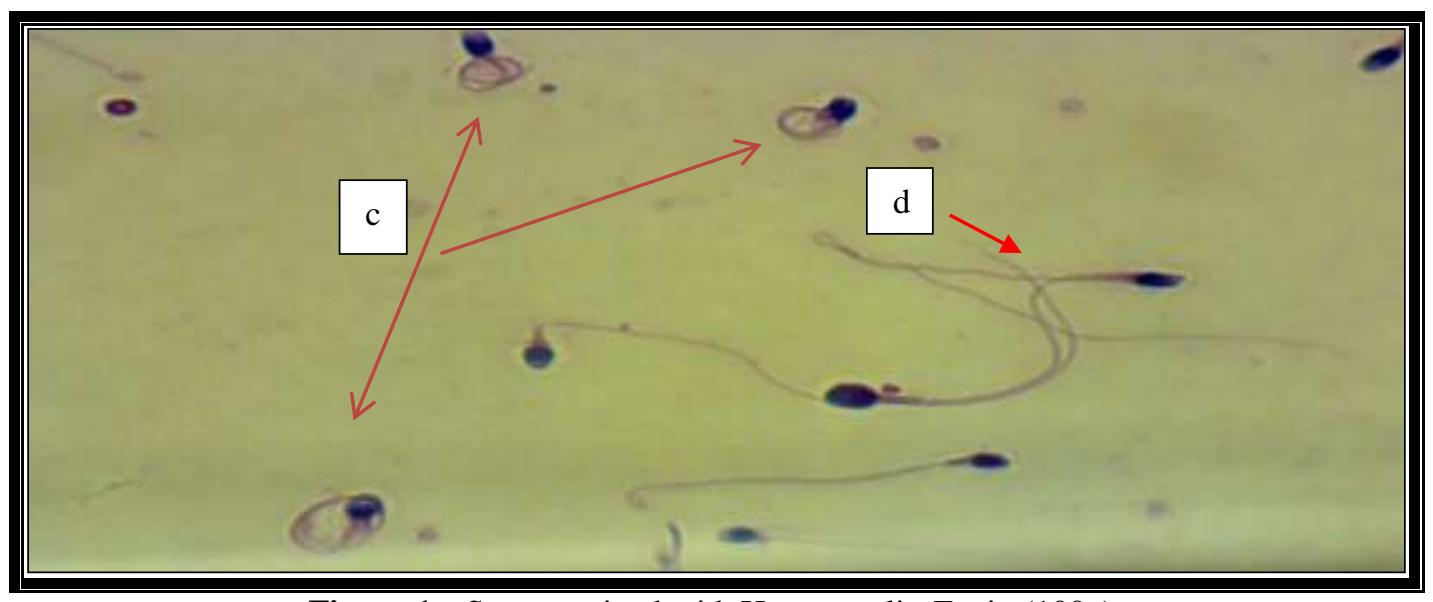

Figure 1:- Sperm stained with Hematoxylin-Eosin (100x):

a-Abnormal, double head c-Abnormal, coiled tail
b-Abnormal, cytoplasmic droplet d- Abnormal amorphous head with double tail.
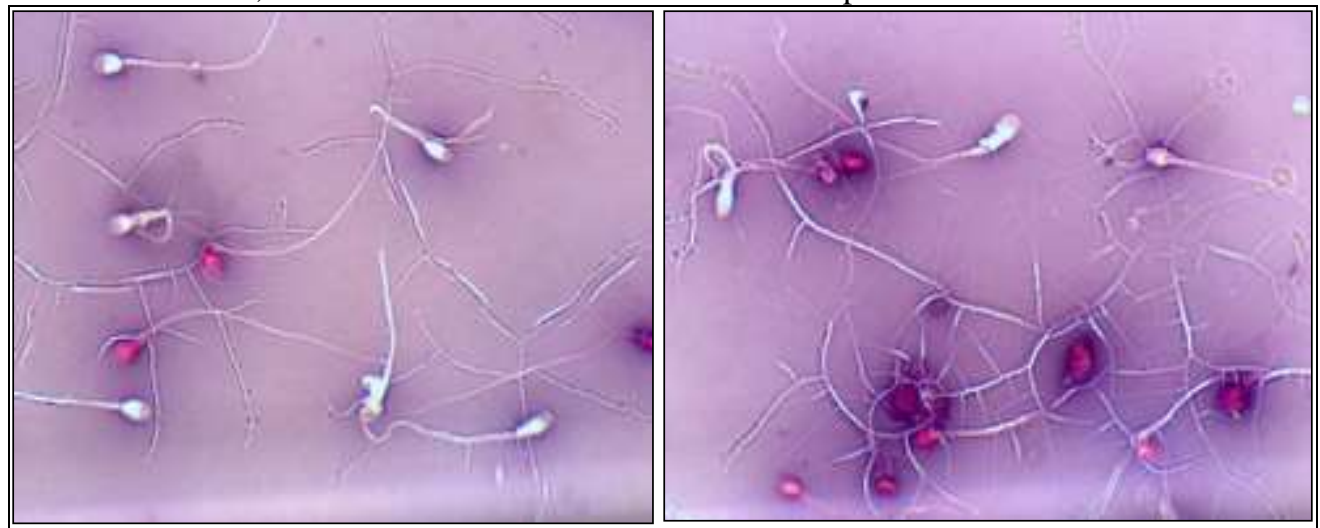

Figure 2:- Eosin-Nigrosin staining(100x): white sperm considered "live" and those that show any pink or red color were considered "dead"

\section{Discussion:-}

Effect of in vitro activation by Free FertiCult medium (control) on certain sperm function parameters:

There was highly significant $(\mathrm{P}<0.001)$ reduction in concentration of spermatozoa following in vitro activation by free FertiCult medium due to the inability of the dead and abnormal sperm with poor motility to swim up and migrate from sperm pellet to the upper layer of culture medium. These results were in agreement with other studies using culture medium for separation and activation of sperm in vitro ${ }^{(19)}$.Regarding sperm motility, there was significant increase in sperm motility compared with pre activation. These cultures contain many ions, and essential nutrients such as sodium, potassium, calcium, magnesium, phosphate, pyruvate and lactate, which works as source of energy to activate sperm, and increases their movement ${ }^{(20)}$. This in agreement with study of (Yahiya $\left.\mathrm{K}\right)^{(21)}$ that describes a significant improvement in sperm motility percentage. There was significant decrease in non-motile sperm (grade D) may be due to that only motile sperm can swim up to the upper layer while dead and immotile sperm remain in the pellet of the medium, and this was consistent with the findings of the (Makler, et al. $)^{(22)}$. Our research found significant $(\mathrm{P}<0.05)$ increment in morphologically normal sperm (MNS) compared with results before activation. That may be because of sperm with normal shapes characterized by their higher activity while sperm with abnormal shapes, characterized by weak motility remain in the bottom ${ }^{(23)}$.

\section{Effect of in vitro activation by Free FertiCult medium (control) and GSH supplemented medium on certain} sperm function parameters before cryopreservation:

A highly significant decrease in sperm concentration compared to before activation due to method of preparation (centrifugation-swim up), after centrifugation sperm migrate toward the opposite effect of gravity, and this is consistent with the findings of (Al-Shimerty, et al.) ${ }^{(23)}$.In current research results showed that there was significant rise in sperm motility percent grade A, and high significant increase in grade B and (grade A+B), this agreed with $(\text { Dhirgam, et al. })^{(24)}$. Glutathione (GSH) presence has been noticed in both intracellular -within the sperm- as well as 
extracellular in seminal plasma, acts as an important endogenous non-enzymatic antioxidant.Chaudhary, et al., found significant negative correlation between GSH and oxidative stress levels in seminal plasma. Therefore, GSH might have some fertility enhancing role by reducing lipid peroxidation, whereas high levels of oxidative stress means poor semen quality. GSH could be used as a chemical marker to assess male fertility by determination of GSH concentrations in seminal plasma ${ }^{(25)}$. Griveau and Le Lannou examined the ability of superoxide dismutase (SOD) and GSH to influence the loss of motility and acrosome reaction rates in spermatozoa prepared by centrifugation. Both GSH and SOD have a protective effect on rates of acrosome reaction and motility ${ }^{(26)}$. Therefore addition of GSH to the medium improves sperm motility in infertile men.

Our results showed that there was a positive effect of GSH when supplemented to the medium, on the percentage of MNS compared with pre-activation and control medium, this is consistent with the findings of (Dhirgam, et al. $)^{(24)}$.Sperm lipid membranes contain a particularly high percentage of poly-unsaturated fatty acids ${ }^{(27)}$. These polyunsaturated fatty acids (PUFAs) give the plasma membrane the fluidity needed to participate in membrane effusion events associated with fertilization. GSH act as an antioxidants that works to control reactive oxygen species and protect sperm from the effect of increased oxidative stress. Bhardwaj, et.al observed that there is positive correlation between sperm concentration, motility and normal sperm morphology with levels of seminal $\mathrm{GSH}^{(28)}$.

Effect of in vitro activation by medium contain Glutathione on cryopreserved human semen:

The results of the present study found a positive effect of GSH addition to the media resulting in enhancing certain sperm function parameters (motility, MNS and vitality) after cryopreservation compared with control medium. This results was consistent with the finding of Ghorbani, et al ${ }^{(29)}$.During thawing, there is an increase in the levels of ROS, with increase in superoxide anion production due to a decrease in SOD activity and to an increase in GSH oxidation by hydrogen peroxide. Decrease intracellular GSH and increased ROS production during cryopreservation could be one of the concomitant causes of low viability in thawed sperm ${ }^{(30)}$. Another study done by (Gadea, et al.) found that GSH content is decreased by freezing and thawing process, and the addition of GSH to the freezing extender could be beneficial in controlling ROS production and improving the function of frozen human spermatozoa ${ }^{(31)}$.

\section{Conclusion:-}

There is positive effect and improvement in certain sperm functional parameters after in vitro sperm activation by GSH supplemented medium, before and after cryopreservation.

\section{References:-}

1. Mohazzab AM, Akhoondi M, Heidari S h, et al. :Fertility preservation in boys and men with cancer, Tehran, Fertility and Infertility Journal. 2011; 12(2): 73-84.

2. David kG, Ariel W, Colin MH, and Zeev S. Text book of Assisted Reproductive Techniques. Laboratory and clinical perspectives, Third edition.Vol(1) UK:publication; 2009.

3. Guzick D, Overstreet JW, Factorlitvak P, et al. Sperm Morphology, Motility, and Concentration in Fertile and Infertile Men. Engl med.2001; 345:1388-1393.

4. Dohle GR, Jungwirth A, Colpi G, Diemer T. Guide line on male infertility. EurAssociatUrol 2007; 11: 8- 21.

5. Marlea D, Nicoletta T, Marco N, et al.: Human Sperm Cryopreservation: Update onTechniques, Effecton DNA Integrity, and Implications for ART. Advances in Urology .2012; 12 pages.

6. Eva M, Andrés J, and James K,: Human Sperm Cryopreservation. European Medical Journal. 2016; 1(1):86-91.

7. Long JA. Avail semen cryopreservation:What are the biological challenges, Poultry science.2006; 85: 232-236.

8. Lushchak VI. Glutathione Homeostasis and Functions: Potential Targets for Medical Interventions, Journal of Amino Acids, Hindawi Publishing Corporation. 2012; 26 pages.

9. Luberda Z. The role of Glutathione in Mammalian gametes. ReprodBiol. 2005; 5: 5-17.

10. Koji A, and Toshio N.Glutathione in Cellular Redox Homeostasis: Association with the Excitatory Amino Acid Carrier 1 (EAAC1).Molecules.2015; 20: 8742-8758.

11. Duan S and Chen C, :S-nitrosylation/denitrosylation and apoptosis of immune cells, Cellular \& Molecular Immunology. 2007; 4(5): 353-358.

12. Halliwell B, and Gutteridge JM, Free Radicals in Biology and Medicine, Clarendon Press, Oxford, UK;1989.

13. Agrawal YP and Vanha-Perttula T. Glutathione, Lglutamic acid and gamma-glutamyltranspeptidase in the bull reproductive tissues. Int J Androl. 1988; 11: 123-131. 
14. Ghorbani M, VatannejadA, Khodadadi I, et al. .Protective Effects of Glutathione Supplementation Against Oxidative Stress During Cryopreservation of Human, US National Library of Medicine. 2012; 37(1): 34-40.

15. Al-Dujaily S S and Al-ani I A. Artificial insemination outcome following washing the cervix and uterus with pentoxifylline. Middle East Fertility Society (MEFs) 4th annual Scientific Congress Egypt-Cairo. 2009.

16. Gil-Salom M, Romero J, Minguez Y, et al. Pregnancy after intracyto-plasmic sperm injection with cryopreserved testicular spermatozoa. Hum. Reprod.1996; 11: 1309-1313.

17. Sonia M and Agarwal A. Aworkbook on human spermatozoa and assisted conception."Chapter16" New Delhi: Jaypee Brothers. 2012: 96-97.

18. Statistical Analysis System, User's Guide. Statistical. SAS.Version $9.1^{\text {th }}$ ed. SAS. Inst. Inc. Cary. N.C. USA. 2016.

19. Allaw AK. Treatment and In Vitro Sperm Activation for Immunologically Infertile Patients M.Sc. Thesis, College of Medicine. KufaUniversity; 1999.

20. Al-Obidy S. Effect of using the FertiCult Flushing medium in sperm parameters activation in vitro infertile patients suffering from Ashthenospermia.M.S.c thesis, College of Sciences, Kufa University; 2010.

21. Yahya K, Al-Sultani S R, Al-Katib S. Effect of vitamin C on in vitro sperm activation of asthenozoospermic infertile patients. American Journal of Research Communication. 2013; 1(10): 40-48.

22. Makler A StollerJ, and Shiran EM. Dynamic aspects concerned with mechanism of separating motile sperm from non-motile sperm, leukocytes, and debris with the use of high - density percoll. Gradients. Fertil. Steril, 1998; 70:961 - 966 .

23. Al-Shimerty DF. In-vitro Human Sperm Activation of Infertile Patients Suffering from Oligozoospermic by using Different Techniques. M.S.c thesis, College of Sciences, Kufa University; 2014.

24. Dhirgam F H. Sperm Activation of OligospermicPatintts at Using Swim-Down Technique and Swim-UP Technique After Centerfucation By FertiCult Flushing Medium with Glutathione. World Journal of Pharmaceutical Research.2017; 6 (7).

25. Chaudhari AR, Piyali D, Ramji S: Study of oxidative stress and reduced glutathione levels in seminal plasma of human subjects with different fertility potential. Biomedical Research .2008; 19 (3): 207-210.

26. Griveau JF and Le Lannou D. Effects of antioxidants on human sperm preparation techniques, Int $J$ of Androl.1994; 17: 225-231.

27. Koppers AJ, Garg ML, Aitken RJ. Stimulation of mitochondrial reactive oxygen species production by unesterified, unsaturated fatty acids in defective human spermatozoa. Free RadicBiol Med .2010; 48: 112-119.

28. Bhardwaj A, Verma A, Majumdar S,et al. Status of Vitamin E and reduced glutathione in semen of Oligozoospermic\&azoospermic patients. Asian J Androl. 2000; 2: 225-228.

29. Ghorbani M, Vatannejad, and Akram; et al.: Protective Effects of Glutathione Supplementation against Oxidative Stress during Cryopreservation of Human Spermatozoa, Cryoletters. 2016; 37, (1): 34-40.

30. Lasso L, Noiles EE, Alvarez JG ,et al. Mechanism of superoxide dismutase loss from human sperm cells during cryopreservation ,Journal of Andrology.1994; $15: 255$.

31. Gadea J, Molla M, Selles E, et al. .Reduced glutathione content in human sperm is decreased after cryopreservation: Effect of the freezing and thawing extender . cryobiology.2011; $62: 40-46$. 